\title{
Volitional Motion Theory
}

\author{
Masaru Kondo*
}

August 14, 2022

\begin{abstract}
I propose a mathematical model for quantifying willpower and an application based on the model. Volitional Motion Theory (VMT) is a mathematical model that draws on classical mechanics, thermodynamics, statistical mechanics, information theory, and philosophy. The resulting numbers are statistical theoretical values deduced using observable data. VMT can be applied to a variety of fields, including behavioral science, behavioral economics, and computational neuroscience. For example, "What is animal spirit in economics?" VMT is one proposal to answer this question. In addition, a scheduling application has been created to validate VMT. This application is open to the public for anyone to use. ${ }^{1}$
\end{abstract}

\section{Introduction}

Initially, we will discuss some important points in proposing this VMT. It seems impossible in the 21st century to propose an exact mathematical model to measure will. In the first place, will is a thing-in-itself [1] that cannot be observed. However, we think that it is also an important element of science to not only observe and understand phenomena, but also to reason, formulate hypotheses and verify them one by one. It is also beneficial if we quantify will and use it rationally. Based on the above assumptions, we propose a VMT.

The VMT proposed in this paper mainly uses the methods of classical mechanics [2], we think that the theory is intuitive and familiar to both children and adults. In addition, we think that familiarity is a very important order of systematization of theories.

\footnotetext{
* paper@thinkupstudio.com

${ }^{1}$ https://onefunc.com
} 


\section{Equation of Volitional Motion}

\subsection{Sensory Mass $(m)$}

Sensory mass is obtained by using the number of experiences in the observation. ${ }^{2}$

\subsubsection{Golden Experience Information $(\phi)$}

$n$ is the number of occurrences in the observation and is a natural number $\left(\mathbb{N}^{+}\right)$.

$$
\begin{gathered}
f_{n}=\frac{1}{\sqrt{5}}\left\{\left(\frac{1+\sqrt{5}}{2}\right)^{n}-\left(\frac{1-\sqrt{5}}{2}\right)^{n}\right\} \\
\phi_{n}=-\log _{2} \frac{1}{f_{n}}
\end{gathered}
$$

\subsubsection{Average Sensory Mass $(\bar{m})$}

$N$ is the number of experiences contained in a single event. ${ }^{3}$

$$
\begin{gathered}
m_{n}=\frac{1}{\phi_{n}} \\
\bar{m}=\frac{1}{N} \sum_{i=1}^{N} m_{i, n}
\end{gathered}
$$

\subsection{Sensory Acceleration $(a)$}

The sensory acceleration is obtained using the sensory mass and the sensory prediction period $(s) . s$ is a subjective estimate of the event duration. ${ }^{4} x$ is the event duration of the actual scheduled. $t$ is the physical time evolution. Subject and Object Synchronization factor (SOS, $\gamma$ ) synchronizes physical and sensory time evolution. $\vec{t}$ is the sensory time converted from sensory velocity to physical time. ${ }^{5} \vec{x}$ is the duration of sensory time.

\footnotetext{
${ }^{2}$ In the verification application, the number of experiences is the number of words used in the schedule's event names. For example, for the event name "feature kickoff meeting", three words are used: "feature", "kickoff', and "meeting".

${ }^{3}$ In this paper, we will denote the average sensory mass $\bar{m}$ by $m$.

${ }^{4}$ Sensory prediction period $(s)$ will be obtained using machine learning and other methods.

${ }^{5} \vec{t}$ has a vector accent. But this does not mean that we are claiming that volitional motion has a direction.
} 


\subsubsection{Sensory Velocity}

$v=\frac{1}{1+\left(1 / v_{t_{0}}-1\right) e^{-m t}}$

2.2.2 Average Sensory Velocity

$\bar{v}=\frac{1}{x} \sum_{t=1}^{x} v_{t}$

\subsection{Will Power}

$W=m a=m \dot{v}$

\subsubsection{Will Momentum}

$u=m v$

\subsubsection{Will Impulse}

$I \Delta t=u_{t}-u_{t_{-1}}$

\subsubsection{Driving Power}

$D=W x$

\subsubsection{Sensory Period}

$x=\sum_{t=1}^{x} v_{t}$

\subsubsection{Sensory Acceleration}

$a=\frac{d v}{d t}$

\subsubsection{SOS and Sensory Time}

$\vec{t}=\gamma_{t, s, v}=\frac{t}{\sqrt{(s-v)^{2}}}$

\subsubsection{Driving Efficiency}

$Q=\frac{\Delta D}{\Delta t}$

2.3.5 Activity

$p=u \vec{t}$

\subsubsection{Total Activity}

$A=u \vec{x}$

\subsubsection{Activity Efficiency}

$$
E=\frac{\Delta A}{\Delta \vec{t}}
$$

\subsubsection{Life Activity}

$$
L=\sum_{i=1}^{n} A_{i}
$$

\subsubsection{Free Activity}

$V=L-A_{i}$

\section{Volitional Space}

Volitional Space is basically a two-dimensional Euclidean space $\left(\mathbb{R}^{2}\right)$. For example, the Euclidean distance between two points can be measured as $d=\sqrt{\left(x_{1}-y_{1}\right)^{2}+\left(\vec{x}_{2}-\vec{y}_{2}\right)^{2}}$. In addition, when converted to the real world by SOS, the naive world $\mathbb{R}^{3}(x, y, z)$ can be said to be $\mathbb{R}^{4}(x, y, z, \vec{t})$. For example, assuming a Lorentz factor $\left(\gamma=\frac{1}{\sqrt{1-v^{2} / c^{2}}}\right)$, the world point is $\vec{t}=\frac{t \gamma}{\sqrt{(\vec{s}-\vec{v})^{2}}}$ and $0=\left(x^{2}+y^{2}+z^{2}\right)-(c t \vec{t})^{2}$.

In addition, we propose the use of parentheses to distinguish between VMT and physical quantities when expressed in mathematical formulas. For example, in the above example, $\vec{t}=\left[\frac{t}{\sqrt{(s-v)^{2}}}\right] \gamma$. 


\section{Situational Motions}

In this section, we propose situational motions. At this stage, situational motions is still experimental and assumes a macroscopically observable range.

\subsection{Forgetting Experience Motion $(\hat{\phi})$}

The number of experiences decays with time. $d$ is the number of days elapsed $\left(\mathbb{N}^{+}\right) .{ }^{6}$

$$
\hat{\phi}_{d}=\frac{1}{\sqrt{5}}\left\{\left(\frac{1+\sqrt{5}}{2}\right)^{d}-\left(\frac{1-\sqrt{5}}{2}\right)^{d}\right\}
$$

$\hat{\phi}_{d}$ is used as a number to determine the golden experience information. $\left(\hat{n}=n-\hat{\phi}_{d}\right)$. As a result, the amount of golden experience information is $f_{\hat{n}}$. If $\hat{n}$ is less than 1 , it is set to 1 .

\subsection{Initial Vibration $(\psi)$}

$a$ is the sensory acceleration.

$$
\begin{gathered}
\omega_{t}=\frac{2 \pi}{a_{t}} \\
\psi_{t}=e^{-(1-m) t} \cos \left(\omega_{(t-1)}\right) \\
v^{\prime}=\sum_{t=1}^{N} v_{t}+\psi_{t}
\end{gathered}
$$

\subsection{Fluctuation of Sensory Velocity $(F)$}

Fluctuations in sensory velocity are added to the sensory velocity.

$$
\begin{aligned}
& F_{m, v, t}=e^{-(1-v) m t} \\
& v^{\prime}=\sum_{t=1}^{N} v_{t}+F_{m, v, t}
\end{aligned}
$$

\footnotetext{
${ }^{6}$ Situational Motions is experimental, but the "act of forgetting" is probably essential for practical purposes. This is because the current equation of volitional motion is a mathematical model of a vacuum state, analogous to classical mechanics. Also, we can't assume a device that can store memories indefinitely, and there are likely to be individual differences.
} 


\subsection{Modify Motion $(\sigma)$}

During motion, the sensory prediction period $(s)$ is probably updated. This update is called a modify motion, and that modify motion is triggered in some way. For example, consider the case where the prediction time $(x)$ is 50 minutes and $s$ is predicted to be 60 minutes. In this case, for example, 30 minutes after the event starts, the subject repredicts the $s$ to be 50 minutes instead of 60 minutes. This is the modify motion.

The triggers for modify motion are thought to occur both actively and passively. Although the definite timing of the modify motion is not strictly known, the following is an example where the timing of the occurrence is fixed. ${ }^{7}$

$x$ is the duration of the event in reality. $s$ is the current sensory prediction time. $s^{\prime}$ is the modified sensory prediction time.

$$
s^{\prime}=\sigma(m, s, x, t)= \begin{cases}\left\lceil\frac{x}{\left.1+e^{-m(t-(s / 2))}\right\rceil}\right. & t>s / 2 \\ \left\lceil\frac{x}{\left.1+e^{-m(t-(s / 2))}\right\rceil}\right. & t>x / 2 \\ s & \text { (otherwise) }\end{cases}
$$

\subsection{Happening Motion $(\mu)$}

During work, unintended events occur, and let the unintended events themselves be $\mu$. In other words, the happening motion is not strictly a motion of the observed object itself. The reason why it needs to be explained as a situational motion is because it is a concept that cannot be avoided in reality. Happening motion occurs at a time that cannot be predicted. Also, since this happening motion represents the phenomenon of loss of concentration if we consider it naively, happening motion forces us to execute the modify motion.

In addition, happening motion is only a conceptual proposal and the mathematical model cannot be described. Since the happening motion is close to the concept of happening in aesthetics [3], it is debatable whether it is appropriate to use external predictions in advance. However, it is possible to use Poisson distribution after obtaining data on the external environment.

\subsection{Other possible situational motions}

In the first place, situational motions is considered to be a quantity of information based on experience. In other words, there is no need to distinguish it from sensory mass. However, just as weight can be

\footnotetext{
${ }^{7}$ This example is just a deterministic corrective exercise. If we want to improve it, we should adjust the range of correction depending on the case, using machine learning and so on.
} 
divided into mass, gravitational force, centrifugal force, etc., it is easier to handle practically if it is divided. Therefore, current VMT distinguishes between motion per se and surrounding circumstances.

Situational motions is experimental. For example, if we can freely add the variable of situational motions, we can explain it in any way we like. For example, in the above situational motions, the amount of information related to colors, appetite and sleep is not expressed.

Theories should only be created in a way that makes them easy to criticize. Also, excessive variables should not be added to ensure consistency. For this reason, it is currently considered to be experimental. However, for verification purposes only, the following situational motions is tentatively used in addition to the above. Its mathematical model is omitted in this paper.
- Diversity Index ${ }^{8}$
- Life Rhythm Index ${ }^{10}$
- Position Index ${ }^{12}$
- Tolerance Rasio ${ }^{9}$
- Weather Index ${ }^{11}$
- Start Time Index ${ }^{13}$

\section{Proof of Concept}

https://onefunc.com

https://geo.itunes.apple.com/app/oneplan/id912230923

The mathematical model described above is being validated in a scheduling application (OnefuncPlan). OnefuncPlan provides a variety of functions that apply VMT.

\subsection{How to express willpower}

OnefuncPlan displays five levels of willpower based on the values of the previous month and the current month. This is because displaying the amount of willpower as a numerical value to the users of the application is likely to increase the psychological load. The criterion for the values is not the willpower $(W)$. The criterion is the total activity $(A)$, and the time lapse $(t)$ is calculated in minutes. By expressing the amount of willpower, users can objectively check their motivation by themselves. This is useful for self-management in remote work.

\footnotetext{
${ }^{8}$ Index of diversity of event categories.

${ }^{9}$ Ratio of unique events.

${ }^{10}$ Index of the interval between the occurrence of an events.

${ }^{11}$ Index of relationship between events and weather.

${ }^{12}$ Index of relationship between events and Location.

${ }^{13}$ Index of relevance of event and start time.
} 


\subsection{Application to Decision Theory}

VMT itself is not a decision theory, but it can be applied. Two examples are given below.

\subsubsection{Automatic schedule adjustment and automatic scheduling}

Automatic voting is a deterministic algorithm. Voting is done on days when the overall activity for the day is less than on other days. Furthermore, we use machine learning and other methods to infer the start time from the event name and prioritize the best candidate date. Also, if a candidate date and time for voting has already been determined, another candidate date and time can be listed automatically.

Automatic scheduling can be realized by an algorithm that applies will activity as well as automatic schedule adjustment. In a nutshell, it is an application of the knapsack problem.

The advantage of using the VMT is that it allows for person-centered automatic scheduling.

\subsubsection{Sharing Experiences}

OnefuncPlan provides a function that allows users to temporarily add other users' experience data to their own experience data. In a nutshell, would be the function of a mentor. For example, when user have just changed jobs, user can share the data of seniors in the workplace and get the best solution for that workplace right away.

\section{Volitional Motion Theory as Natural Philosophy}

\subsection{Overall framework}

I will now describe a discussion of VMT, and in the discussion there are terms of philosophy. However, in some cases, the meaning of these terms is used in a different way from the general philosophical terms. Those terms is used only as a term in VMT.

VMT is a concept, an attempt to infer thing-in-itself from representations. Will in VMT is the position that will exists, although it cannot be directly observed. In other words, VMT is purely a tool and does not strictly define the will. These are things we wrote in the introduction, but we will emphasize them again.

\subsection{Stance of VMT}

One of the best features of human reason is its ability to accumulate methods that are realistically effective. Tools and concepts have accelerated the civilization of society. In modern times, even in the microscopic quantum age, where position and momentum cannot be observed simultaneously, people use 
quantum mechanics as a tool. People use these methods because they are reproducible and approximate reality.

People use tools to expand their possibilities. Furthermore, regardless of positivism or instrumentalism, reproducible things convince many people. The simple and timeless means of communication that can convince many people are number and structure. Therefore, VMT is considered on these two principles.

- VMT is purely a tool and does not strictly define the will.

- VMT is expressed in terms of numbers and structures for reproducibility.

Theories should use computational models that approximate reality as closely as possible, and should also be available as tools.

\subsection{The Existence of Will and Representation}

The world is my representation [5]. Furthermore, world are impermanent. If will exists, then representation exists, and if representation exists, then will exists. Everything is a dissipative structure, and the interaction shapes will and representation.

The following are the elements necessary for using the VMT.

- Will. (thing-in-itself)

- Will and Representation. (Lifeworld)

- Object of observation. (Object of observation)

- Observer. (VMT)

- Structure. (Represented data)

- Persistence. (the environment in which an observer can observe. In other words, an environment in which the motion can be sustained.)

Subjectivity and intuition are representations when they are observable. Intuition is generally considered to be the thing-in-itself, and to capture things without reasoning, but even if it is subjective or intuitive, it is a representation when it is recognized.

This does not mean that subjectivity and intuition do not exist in a general sense, but rather that they exist as representations when we recognize them. Will is something that cannot be observed even if we trace it back to the source of perception, subjectivity or intuition.

When we recognize that there is a will, it is not a will. it is representation. It is not possible to actively perceive will itself. In other words, we cannot say, "I have a will". Therefore, the VMT is observed and 
predicted by a third party. In the 21st century, we have computers. The calculation is based on the observed range of "done or not done" rather than "tried."

VMT is only an inference of willpower from observed representations, and will is something that emerges from observed motion. The numerical value of willpower itself does not represent will. The result of body and action is not will. The will itself cannot be objectified.

To summarize, if $L$ as the Lifeworld, $R$ as the representation, and $W$ as the will, then $L=\{R, W\}$ and $W$ is deduced from $W^{\complement}$. The reason why photon behaves as photon is unobservable. The same is true of will.

VMT assumes non-summation, and considers sum of elements $(U)+$ observer $(O)=$ universal system $(U S)$. The observer is the information control mechanism, the VMT itself. Furthermore, the observer $O$ is not included in the whole set. Again, we cannot say "I have a will". ${ }^{14}$

\subsection{Nature of the observer}

If the material used by the carpenter is wood or sculptural bronze, and the result is a building, the material of will is cognition, that manifests itself as movement. ${ }^{15}$

In general, the past is remembered and known, but the future is unknown and can only be predicted. However, the VMT can predict the past, present, and future. This is because VMT is an inference based on observations by a third party.

In addition, the VMT can be used not only to observe people, but also to observe plants. This is not a joke. VMT is only a tool, and it can infer the willpower of any object, letters, pencils, books, or robots. In other words, the VMT has the characteristic of representing the will of all possible objects of observation. This is due to the nature of the observer, not the nature of the observed object.

\footnotetext{
${ }^{14}$ The problem with VMT is that it does not measure the practical value of willpower. VMT is a theoretical value and only assumes its usefulness as a tool. In order to solve this problem, it is necessary to define the existence of some physical energy that connects and correlates representations and volitional motion. This is not explicitly stated in this paper.

${ }^{15}$ Stoics, modification of Epictetus.
} 


\section{About sensory mass}

\subsection{Cognitive Entropy $(\Omega)$}

In VMT, we use the name "mass" because it is expressed using a mathematical model of physics, but it would be more appropriate to express it as cognitive entropy or cognitive dimension.

The fractal dimension $(d)$ is the total number of $1 / a$ contained in $\mathrm{b}[6]$.

$$
\begin{gathered}
a^{d}=b \\
d \log a=\log b \\
d=\frac{\log b}{\log a}
\end{gathered}
$$

Sensory mass is the amount of golden experience information $(\phi)$ that assumes self-similarity $(1 / f)$ through learning. ${ }^{16}$ Also, referring to the fractal dimension, all spatial quantities are $t$.

$$
\begin{gathered}
f^{d}=t \\
d=\frac{\log t}{\log f}
\end{gathered}
$$

The above $t$ is the cognitive space that satisfies all spaces and is represented by $\delta$. The $\delta$ is a sequence of two patterns of representational motion, recognizable and unrecognizable, per time evolution. In other words, it can be considered as the amount of information [8]. The resulting relationship is as follows .

$$
m=\Omega=\frac{1}{\phi}=\frac{-\log _{2} 1 / 2}{-\log _{2} 1 / f}=\frac{\delta}{\phi}=\frac{t}{\phi}
$$

The cognitive space is the derivative of the sensory velocity, that is also the sensory acceleration.

Thus far in this paper, we have explained it in the classical dynamical form $F=m a$, but the correct expression of the relation is as follows.

$$
W=m a=\frac{a}{\phi}=\frac{\dot{v}}{\phi}
$$

In other words, the sensory mass $(m=1 / \phi)$ used in this paper can be said to be the willpower in the case of sensory acceleration 1.

In summary, willpower is a statistic that satisfies or extends $a$ the cognitive space with a self-similar structure. In mechanical terms, willpower can be defined as a force that can produce a sensory acceleration of $a$ on a material point with an information density of $1 / \phi$.

\footnotetext{
${ }^{16}$ This is an assumption based on the topological index. [7].
} 


\subsection{Observation range}

The explanation in this paper is obtained from the number of words in the event name using the verification application as an example. However, it does not mean that this method has to be used.

For example, suppose an observer is looking at a bunch of cars at an intersection in a city. While observing, observer blinks and suddenly all the cars turn into elephants. In this case, for the observer, there is a noticeable difference in perception. This difference depends on the amount of experience, and the amount of experience depends on the range of observation. The car example assumes vision, but all interactions, including auditory, are within the scope of VMT. ${ }^{17}$

\section{About sensory velocity}

\subsection{Relationship between sensory velocity and the Lifeworld}

The basic idea of sensory velocity $(v)$ is a model that depends on how much roughness exists in the perception during a motion of $1 t$. Roughness is the amount of difference or novelty from past knowledge and experience, or in other words, the amount of cognition. In VMT, sensory mass is used as a variable in the mathematical model. ${ }^{18}$

$$
v=\frac{1}{1+\left(1 / v_{t_{0}}-1\right) e^{-m t}}
$$

The structure of the sensory velocity has a structure that converges to 1 . This motion is called Cognistasis. ${ }^{1920}$ Furthermore, the main properties that can be read from this structure are as follows.

- When the sensory mass is large, such as in a first time event, the sensory velocity converges rapidly to 1

- When the sensory mass is small, such as in a multiple experiences event, the sensory velocity gradually converges to 1

In other words, the following.

- If the sensory mass is large, it is in a state of high stimulation and is focused on the Lifeworld.

\footnotetext{
${ }^{17}$ If we consider the example of the verification application strictly, we can consider it as the will of the schedule written by the use rather than the will of the user himself.

${ }^{18}$ Sensory velocity can be thought of as "Pure Velocity". In philosophy and metaphysics, we tend to use such an expression.

${ }^{19}$ Sensory velocity is also cognitive space.

${ }^{20}$ Meaning similar to conatus, derived from natural philosophy.
} 
- If the sensory mass is small, the person is in a state of low stimulation and is not focused on the Lifeworld.

The first time we experience an event, our sensory mass is large, so there is a lot of stimulation, and we concentrate on the event at hand. After that, each time we experience the event, our sensory mass changes and the stimulation decreases, making it harder to concentrate. ${ }^{21}$

\subsection{Subject and Object Synchronization (SOS)}

Sensory velocity is the subjective rate of perception and is different from the physical movement of time. We need something to connect these together, which is represented by the SOS. Let time evolution be $t$ and sensory time be $\vec{t}^{22}$

$$
\begin{aligned}
(s \vec{t})^{2} & =(v \vec{t})^{2}+(t)^{2} \\
\vec{t} & =\frac{1}{\sqrt{(s-v)^{2}}} t
\end{aligned}
$$

For example, if the expected time to bring a cup from the next room is 10 and the actual time taken is 20 seconds, then approximately $\vec{t}=1.05$.

\section{Law of Volitional Motion}

The following is a summary of the above.

\subsection{Observation range dependence principle}

Willpower is a numerical value required by the observer

\subsection{Law of cognitive motion}

The will is always in motion, moving to fill the cognitive space.

\subsection{Volitional Motion Equation}

The mathematical model that satisfies the laws of cognitive motion is the equation of volitional motion.

\footnotetext{
${ }^{21}$ This kind of structure is easy to accept in the context of naive empiricism.

${ }^{22}$ Sensory time can be thought of as "Pure Time".
} 


\section{Application Examples}

\section{1 behavioral economics}

VMT can be applied to expected utility theory [4].

$$
\begin{gathered}
a_{i}=T-\left(T-A_{i}\right) \\
E=\sum_{i=0}^{n} g_{i} a_{i}
\end{gathered}
$$

$E$ is the volitional expected utility, $n$ is the sum of the alternatives, $g$ is the gain, $T$ is the sum of the will activity of all alternatives, and $A$ is the will activity of the alternatives. For example, there are two choices, $i_{0}$ and $i_{1}$. If $A$ of $i_{0}$ is 3 and the gain is 10 , and $A$ of $i_{1}$ is 2 and the gain is 5 , then $i_{0}$ has higher utility.

$$
\begin{aligned}
& i_{0}=10 \cdot 2=20 \\
& i_{1}=5 \cdot 3=15
\end{aligned}
$$

Then, the efficiency of these alternatives for a will activity of 1 is $35 / 5=7$.

We can also consider a more naive method of adding probabilities. $p$ is the probability of occurrence.

$$
E=\sum_{i=0}^{n} p_{i} g_{i} a_{i}
$$

\section{Willpower convergence}

The typical lifestyle in the first half of the 21 st century does not involve working in different fields every day and having multiple homes. If we continue with that constant lifestyle, our overall will activity will be low. ${ }^{23}$

\section{Willpower attraction and Social willpower}

As a result of the willpower convergence effect, volitional motions are more likely to propagate with their neighbors. For example, acquaintances of entrepreneurs are more likely to become entrepreneurs. This means that "starting a business" and "learning to start a business" are not the same thing, but they do affect the volitional motions.

\footnotetext{
${ }^{23}$ It is possible that volitional motions tend to be more active in free and leisure time. In other words, history is made at night.
} 
This phenomenon of propagation and entangling of wills is good if the effect is positive, but negative examples are also possible. For example, the knowledge of a particular school of thought is likely to be biased because it gathers evidence that strengthens its argument. As a result, the will for that school of thought will converge. If we assume VMT, we will not listen to different opinions, and if we do not change our behavior, the total-will-activity will not increase. This characteristic is also true in societies and organizations. In other words, there is no evolution in a completely conservative organization. ${ }^{24}$

The above examples were used in a negative sense, but they can also have a positive meaning. For example, conservative organizations, represented by bureaucrats, also have the role of conservation, and formal rationality [9] also increases predictability, making the organization more stable. In addition, the dialectical method is based on the existence of an axis of opposition, and conservative organizations are likely to be adopted as the axis of opposition. In other words, a conservative organization exists not only for itself, but also for other organizations. ${ }^{25}$

In this section we will discuss propagation of will and collective willpower. Because this is what can easily lead to totalitarianism. I would venture to say that, in most cases, dialectic is useful in strengthening individuals, but is not recommended for use in groups or organizations. People have bounded rationality and limited cognitive abilities [11]. In addition, formal rationality and bureaucracy are local optimizations, and when viewed as a whole, errors exist and possibilities are discarded. ${ }^{2627}$

Individuals and organizations can prevent the convergence of wills by daring to act differently from others, and VMT is a tool that makes it possible to adjust that balance from the observer's perspective.

Finally, to find the collective will, we can simply calculate the willpower and will activity of all the agents in the group. Collective willpower can be used in many fields such as social engineering and economics. For example, collective willpower is thought to be related to the inflation rate, as in the Phillips curve. ${ }^{28}$

\subsection{Demonstration of Attraction Effect}

I created a hypothetical mathematical model by referring to the Kuramoto model [12] and performed computer simulations. ${ }^{29}$ However, as we have explained, the current VMT is a deterministic model.

\footnotetext{
${ }^{24}$ The so-called "corporate bureaucracy" may be the result of a convergence of willpower.

${ }^{25}$ More books about beaurocracy[10].

${ }^{26}$ However, I think that if someone finds the dialectic useful, they should use it.

${ }^{27}$ Convergence of wills requires a period of time in which events are repeated many times. In other words, it is not something that converges in an instant.

${ }^{28}$ There is no data or other evidence of this in this paper. Conjecture read from the structure of the theory.

${ }^{29}$ This is a hypothetical model in which each oscillator has a certain rhythm. Oscillators in a verification application are scheduled events.
} 
Therefore, we get the natural result. ${ }^{3031}$

\section{Problems and issues, etc.}

VMT has a lot of challenges at the moment. some of which are described below.

\subsection{Verification method}

VMT has reproducible. However, this reproducibility is a matter of course. This is because the basic structure of VMT is a statistical value over a range of data. Also, as far as we can confirm with the validation application, it is properly represented. In a nutshell, it works.

\subsection{Types of willpower}

This paper proposes a model that relies on experience, but does not deny a priori will. This is because, for example, the reflex to pain is not only experience.

\subsection{Volitional Spaces as Manifold}

The Volitional Space is a space for each observer. In other words, it can be thought of as a manifold. Manifold is also caused by differences in observers and observation ranges, and is related to social volitional motion. In other words, it is possible to describe a mathematical model based on the differences in volitional spaces. However, at this stage, we do not think that this is necessary, For this reason, we are not making any assumptions at this stage.

\subsection{Are sensory mass and sensory acceleration reasonable values?}

Sensory mass has not been able to quantify its exact quality. For example, person $A$ is afraid of roller coasters, and does not want to ride them a second time, while person B finds them enjoyable. That meaning and quality of their experiences are different, but if they have only ridden the roller coaster once, then $A$ and $B$ are expected to have the same amount of willpower.

Quality is not the same as so-called naive physics. It can be considered as meaning making. Multiple methods of quality of information have been proposed from various perspectives and fields, for example, the concept of logical depth [13], but there is no appropriate one. At this point, we have not speculated on this issue at all, because it seems that no one can solve this problem today.

\footnotetext{
${ }^{30}$ I have not confirmed this model by implementing it in society.

${ }^{31}$ Another possibility is to use the situation motion as a variable and express it using simultaneous ordinary differential equations. In this case, we can consider a model that does not converge. It can also be deterministic chaos.
} 


\subsection{Duration and transition of cognition}

Strictly speaking, $\Omega=a / \phi$ is updated at every time evolution. Therefore, we cannot assume that the cognition is sustained in scheduled event units as in the verification application. In other words, instead of expressing duration of cognition only in terms of sensory velocity, we need to express changes in sensory mass at the same time. For example, in the case of the "go for a walk" event, not only sensory velocity but also more detailed changes in sensory mass are occurring during the walk.

However, the duration and transition of cognition will not be mentioned further. The current VMT is a macroscopic mathematical model, and it considers the transition of cognition only as a situational motions.

\subsection{Application to robotics}

"Why can a person use a new cup?" To put it more abstractly, "why do people appear to be able to perform generic actions?". I think that this can be mimicked with VMT.

In this paper, experience was treated as a single database. However, when assuming human will, it is more realistic to divide it into "physical experience" and "conceptual experience," and further divide these two into short, medium, and long term, for a total of six databases. ${ }^{32}$

\subsection{Willpower and Energy}

The VMT describes willpower by referring to mathematical models in physics, but we will need to discuss the relationship between willpower and matter. In other words, the exact relationship between will and representation. If the theory is extended with these definitions unclear, there is a high possibility that it will become a statistical number game. ${ }^{33}$

However, willpower can be assumed to be entropy, which can be interpreted as $S=k \ln W$. Furthermore, the structure of the cognitive entropy $(\Omega=a / \phi)$ can be interpreted as equivalent to the structure of the thermodynamic entropy $(\Delta S=\Delta Q / T)$. In a word, will is heat. This means that the information control structure is equivalent to the process of Maxwell's demon [14], and this structure obeys the laws of thermodynamics. In other words, VMT is the devil. I can then assume that VMT will be considered from the perspective of information thermodynamics.

But, of course, these are assumptions. In the first place, the structure of the VMT is based on various existing fields, and it is natural that the structure is similar.

\footnotetext{
${ }^{32}$ This is an example. There are many other possible methods.

${ }^{33}$ For me, that's fine. As it stands, VMT is still an interesting toy.
} 


\section{Conclusion}

In this paper, we proposed a theory for inferring willpower with a mathematical model. The equation of volitional motion can be described by following the familiar classical mechanics, which is simple and easy to understand, and it is easy to use practically. In addition, the VMT can be applied to various fields, so there is room for further development.

The VMT can also be read as a theory that mixes natural philosophy and science. This is because it seems impossible to make a proposal like the one in this paper without taking the position that "Magicians" [15]. Some people may not be comfortable, but from a positive point of view, it is modern natural philosophy, and it is one proposal for expanding science.

Furthermore, VMT can be used to realize a human-centered society. This is because if existing capitalism is "superficial capitalism" that acts in accordance with social forms and funds, VMT can realize "real capitalism" based on human will.

\section{References}

[1] Immanuel Kant, "Critique of Pure Reason" 1781

[2] Sir Isaac Newton, "Philosophiae Naturalis Principia Mathematica" 1687

[3] John Cage, "Silence" 1961

[4] John von Neumann, Oskar Morgenstern, "Theory of Games and Economic Behavior" 1944

[5] Arthur Schopenhauer, "The World as Will and Representation" 1819

[6] Benoit Mandelbrot, "The fractal geometry of nature" 1982

[7] Hosoya Haruo, "Topological index" 1971

[8] Claude E. Shannon, Warren Weaver, "The Mathematical Theory of Communication" 1949

[9] Max Weber, "Economy and Society" 1921

[10] Robert King Merton, "Social Theory and Social Structure" 1949

[11] Herbert Alexander Simon, "Administrative Behavior" 1947

[12] Kuramoto Yoshiki, "Self-entrainment of a population of coupled non-linear oscillators" 1975

[13] Charles Henry Bennett, "Logical Depth and Physical Complexity" 1988 
[14] Charles Henry Bennett, "Demons, engines and the second law" 1987

[15] John Maynard Keynes, "Newton the Man" 1946 